\title{
A Printed, Dry Electrode Frank Configuration Vest for Ambulatory Vectorcardiographic Monitoring
}

Gordon Paul, Russel Torah, Steve Beeby and John Tudor*

Abstract - This paper describes the design and fabrication of a screen printed network of bio-potential measurement electrodes on a garment, in this case a vest. The electrodes are placed according to the Frank configuration, which allows monitoring of the electrical behavior of the heart in three spatial orientations. The vest is designed to provide stable contact pressure on the electrodes. The electrodes are fabricated from stencil printed carbon loaded rubber and are connected by screen printed silver polymer conductive tracks to an array of vias, which form an electrical connection to the other side of the textile. The vest is tested and compared to Frank configuration recordings that were obtained using standard self-adhesive ECG electrodes. The vest was successfully used to obtain Frank configuration recordings with minimal baseline drift. The vest is fabricated using only technologies found in standard textile production lines and can be used with a reduced setup effort compared to clinical 12-lead examinations.

Index Terms-Electrocardiogram, wearable, screen printing, smart fabrics

THE electrocardiogram (ECG) is a vital tool in the diagnosis

This work was partially funded by the Bravehealth project (Patient Centric Approach for an Integrated, Adaptive, Context Aware Remote Diagnosis and Management of Cardiovascular Diseases, FP7-ICT-2009-4) as part of EU framework package 7. Thanks go to Andrea Vitali of ST Microelectronics for helpful suggestions.

Gordon Paul is now at the School of Engineering and Material Science, Queen Mary University of London, London, E1 4NS, United Kingdom.
24 of heart conditions. Some symptoms can be recognized with

25 a single lead, which provides a view of the heart in only one

26 dimension. This is achieved by comparing two points on the

27 skin surface using skin contact electrodes. An examination

28 with greater detail can be achieved by measuring the skin

29 surface voltage from several directions, providing a three-

30 dimensional view of the heart's behavior [1].

31 Examinations of this type are usually carried out in a 32 hospital setting using self-adhesive silver/silver chloride $33(\mathrm{Ag} / \mathrm{AgCl})$ electrodes. The 12-lead system is typically used.

34 This is obtained using an electrode on each arm and leg and 35 six across the front of the chest. However, this requires 36 significant training and setup time for correct placement of 37 the electrodes and the electrodes usually cannot be re-used 38 [2]. A Frank configuration electrocardiogram, using only 8 39 electrodes, provides the same information as a 12 lead 40 examination. Research has been carried out on extrapolating 41 the 12-lead data, with which physicians are trained, from the 42 Frank configuration ECG [3]. It has been shown that 43 transformations from such reduced lead systems are 44 clinically accurate when the signal to noise ratio is

Russel Torah, Steve Beeby and John Tudor are in the School of Electronics and Computer Science, Faculty of Physical Sciences and Engineering, University of Southampton, Southampton, SO17 1BJ, United Kingdom. *Corresponding author email: mjt@ecs.soton.ac.uk.

All data supporting this study are openly available from the University of Southampton repository at http://doi.org/10.5258/SOTON/404548 
61 failure [10,11].

71 be worn by an ambulatory patient for a fixed period of, for

72 example, one week, facilitates a more detailed and accurate

73 diagnosis of cardiac events. Some such events occur 74 infrequently and are therefore unlikely to occur when a
75 patient is in a clinical setting undergoing a multiple lead 76 examination.

77 A Frank configuration monitoring vest is fabricated here 78 using screen and stencil printing to create an insulated, 79 conductive network on a vest that connects dry, passive, 80 conductive rubber electrodes to a centralized set of 81 conductive vias. The Frank configuration electrode position 82 system is used because this system gives a full three83 dimensional view of the heart with only seven differential 84 electrodes and a reference electrode. The resulting system is 85 examined with table-top analogue amplifiers and data 86 acquisition systems. Portable electronics and data 87 transmission are outside of the scope of this paper.

\section{VEST DESIGN}

89 In Frank's original design [12] there was an electrode 90 placed on the neck and on the foot. For an easily wearable

91 monitoring garment these electrode positions must be moved 92 on to the torso, so that all electrodes can be placed on single 93 garment, in this case, a vest. The revised electrode positions, 94 as defined by the BRAVEHEALTH project [13] from which

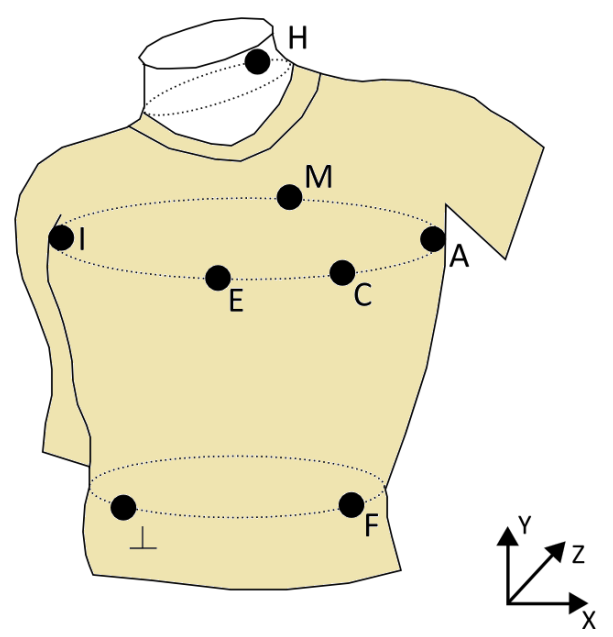

Fig. 1. The Frank configuration vest concept design from the BRAVEHEALTH project. 
this research originated, are shown in Fig. 1. Electrode F is moved up from the foot and electrode $\mathrm{H}$ is moved down from the neck.

A conductive network was designed to connect electrodes in the Frank configuration positions to vias at a centralized point on the vest. From this centralized point the electrodes can be connected to an external instrumentation amplifier with a single set of cables. In future, amplification and wireless communication electronics can be integrated into this central point so that cables are not required.

Screen printing is used to fabricate a network that is composed of electrode pads, centralized via contact pads and the conductive tracks that connect between the electrodes and the vias. Dry passive electrodes are used here to simplify fabrication, however this technology is compatible with dry active electrodes described previously $[14,15]$. Because the maximum printable area is limited for the equipment used,

the printed network is fabricated with two separate designs

that are printed individually, as shown by the two highlighted areas in Fig. 2. The printed textiles are sewn to a vest textile with silicone foam inserted between the printed and vest textiles, as recommended by Ottenbacher et al [16], to improve contact pressure stability. The vest textile then has

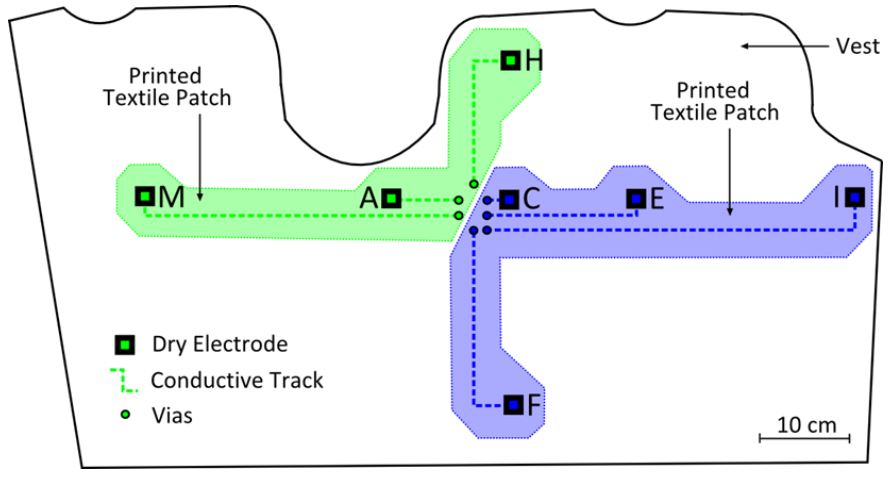

Fig. 2. A concept diagram showing the positions of electrodes, conductive tracks and vias on the textile vest from the inside.

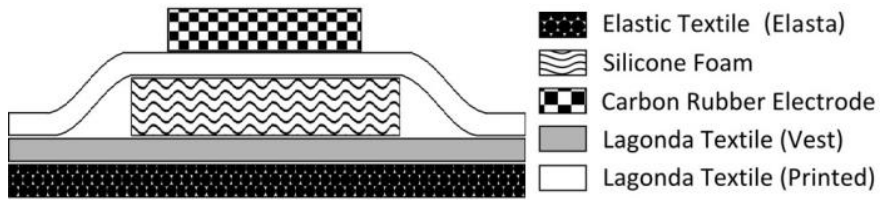

Fig. 3. The layer structure at the site of an electrode. The electrical connection to the electrode is not shown.

118 elastic strips sewn to the opposite side to improve form

119 fitting, ensuring the electrodes make stable skin contact. A

120 diagram of the layer structure at an electrode site is shown in

121 Fig. 3. The electrical connection to the electrode is not

122 shown

\section{FABRICATION METHODS}

124 Since the conductive network is screen printed on to a 125 woven textile, an interface paste is used to reduce the surface 126 roughness of the textile. This interface is screen printed 127 directly on to the textile and is polyurethane based. Using this 128 interface, a low-resistance network of conductive tracks can 129 be fabricated on the textile using a thinner layer of silver 130 polymer paste than would be possible without the interface.

131 This conductive network is then insulated with the same 132 polyurethane paste as used for the textile interface. This 133 methodology was first described by Yang et al [17]. 
The network is screen printed on to a large square $(50 \times 50$

135

$\mathrm{cm}$ ) of a woven textile, Lagonda, supplied by Klopman International S.R.L. This textile was selected because it is commonly used by the supplier in medical clothing and provides a good compromise between comfort and suitability for printing. The yarns of this textile are composed of cotton, polyester and Lycra and the textile thickness is $290 \mu \mathrm{m}$. The screens used have a mesh density of 250/inch and a mesh angle of $45^{\circ}$, with the emulsion thickness varying depending on the required layer thickness. First, FabInks-UV-1004 [18] interface paste is printed and cured several times using a screen with emulsion thickness $30 \mu \mathrm{m}$. This creates a smooth interface layer on the textile, with a thickness around $100 \mu \mathrm{m}$ above the textile surface. Then, Fabinks TC-C4001 silver conductor is printed using a screen with emulsion thickness $5 \mu \mathrm{m}$, resulting in a layer $5-10 \mu \mathrm{m}$ thick. This is cured in an oven at $120{ }^{\circ} \mathrm{C}$ for 10 minutes. Finally, the conductive tracks are encapsulated with a further two print-cure cycles using FabInks-UV-1004 and a screen emulsion thickness of $30 \mu \mathrm{m}$, providing an encapsulation layer of thickness $60 \mu \mathrm{m}$. The textile patches are then cut out from the larger square of textile. The screen designs are shown in Fig. 4 and the printed textile patches are shown in Fig. 5. The resistance of each silver track was measured with a Tenma 72-7735 digital multi-meter and the measured resistances are marked on Fig. 5. The resistance of each conductive track is measured from the central point of the via pad to the central point of the electrode pad.

The electrode pads are then stencil printed with carbon black loaded silicone rubber. This material is selected

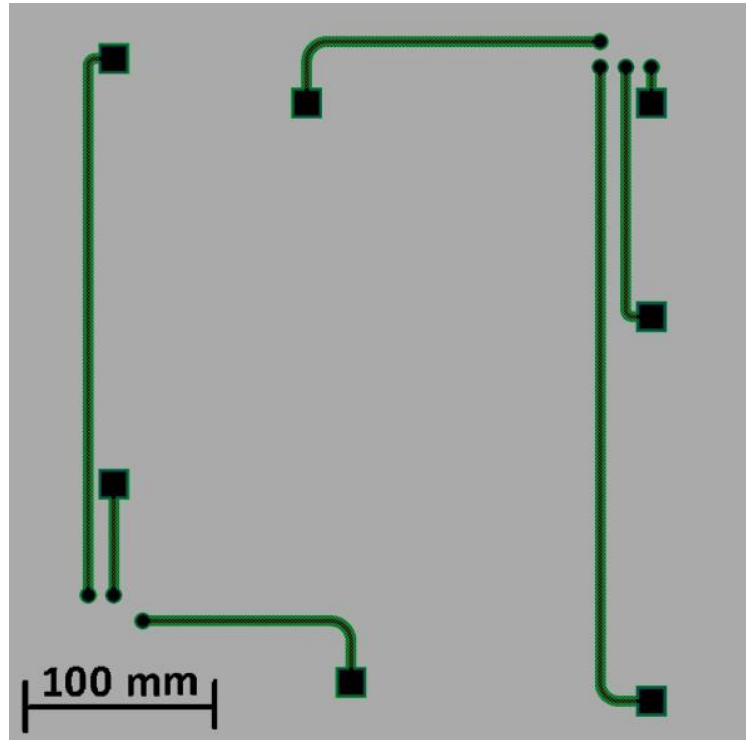

Fig. 4. The screen design for the Frank configuration monitoring vest.

164 because it provides a high level of flexibility for a conductive 165 material, while being water resistant and having a low 166 surface energy, which prevents hair and dirt gathering on the 167 electrode and facilitates cleaning. It can also be molded into 168 different shapes and consequently an uneven skin contacting 169 surface can be created, which allows the electrode to make 170 contact more easily when the skin is hairy. An aluminium

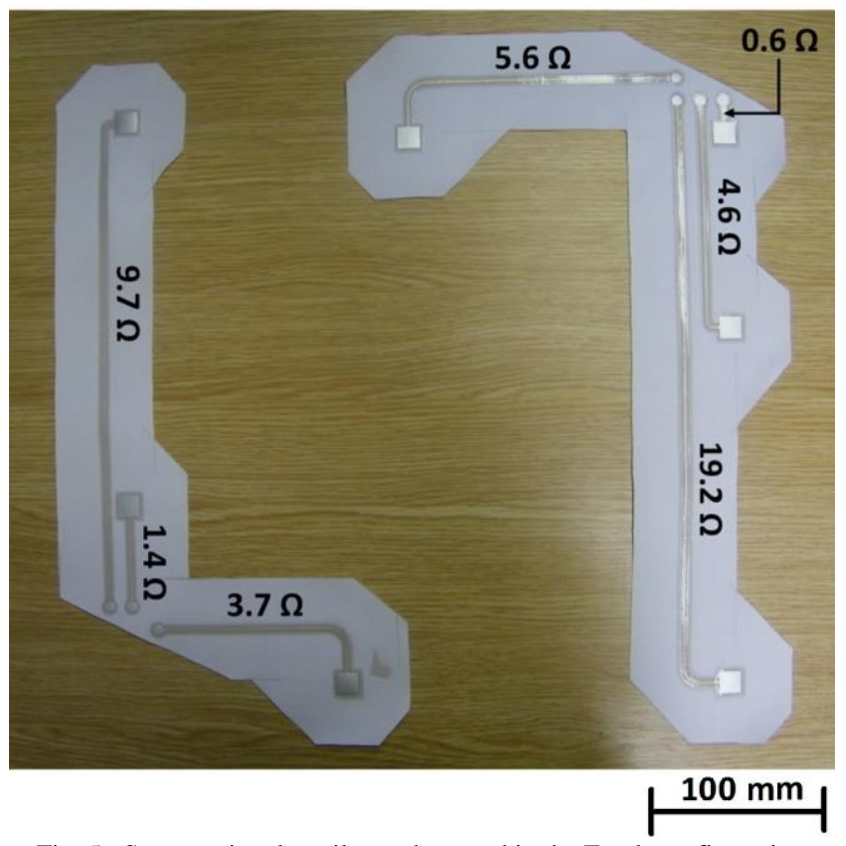

Fig. 5. Screen printed textile patches used in the Frank configuration monitoring vest and associated track resistance values. 
171

172 stencil with a thickness of $3 \mathrm{~mm}$ is used. The stencil printed

carbon loaded rubber, forming the skin contact area of each electrode, has a thickness of $3 \mathrm{~mm}$ and a length and width of 26 and $22 \mathrm{~mm}$ respectively. The screen and stencil printed textile patches are shown in Fig. 6.

The printed textile patches are then sewn to a vest-shaped textile. Silicone foam with thickness $6.3 \mathrm{~mm}$ is placed

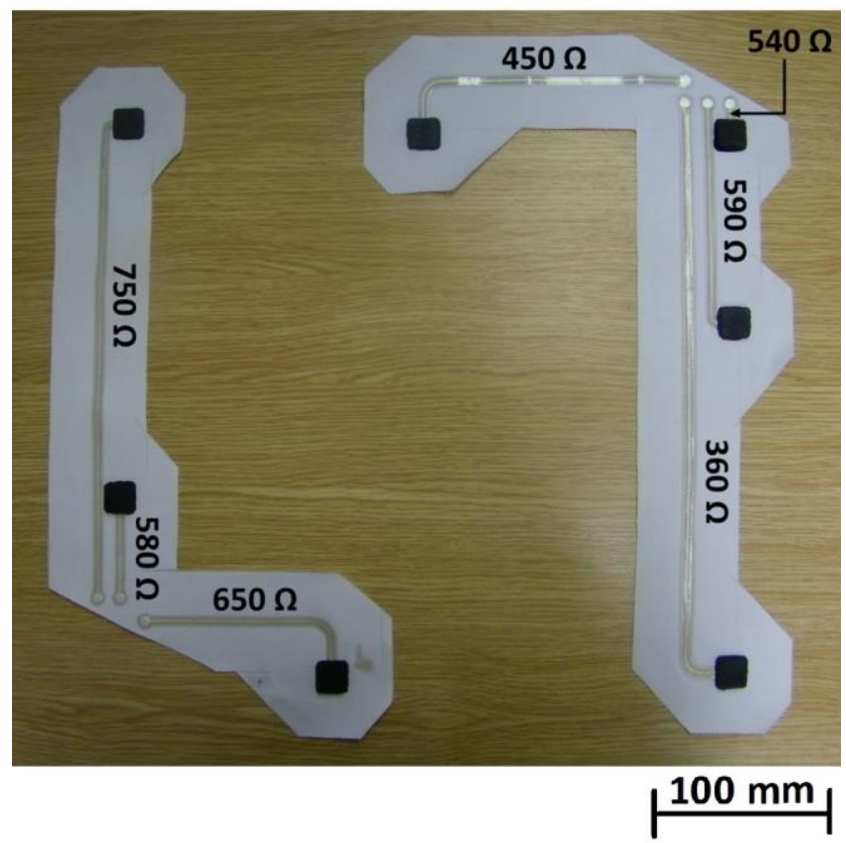

Fig. 6 . The screen printed textile patches with stencil printed carbon black loaded silicone rubber electrodes and associated track resistance values after

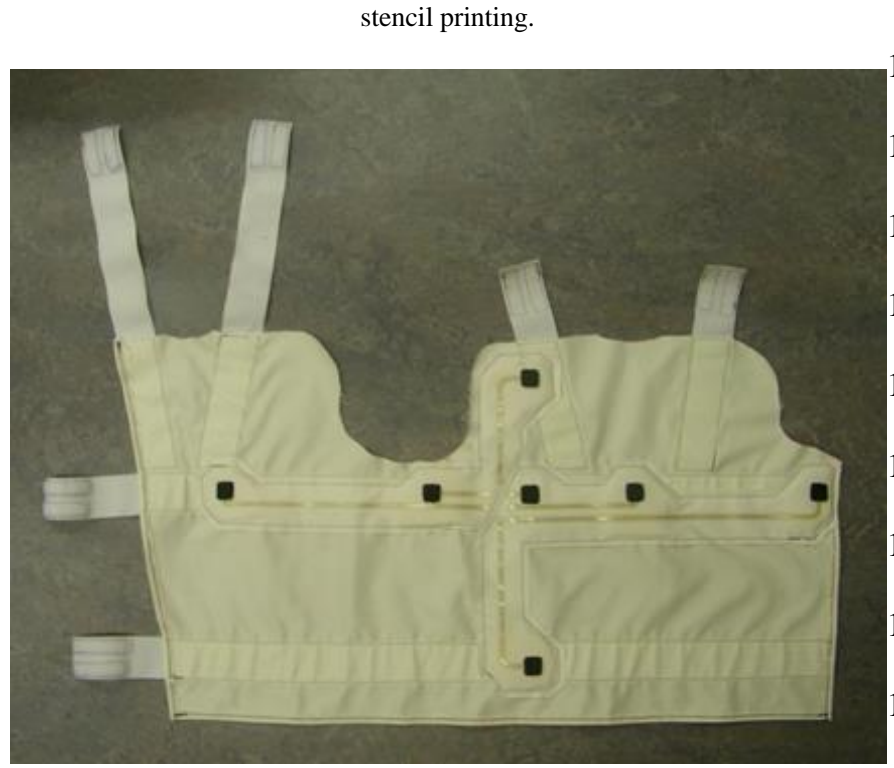

188

189 via pads in the centralized array. This allows electrical

190 connection through the textile so that electronics can be

191 added on the outer, non-printed side of the vest without

192 discomfort when the vest is worn. These steel buttons have

193 short wires attached that connect to the amplifier via a

194 resistive network. The fabrication process for similar stencil

195 printed electrodes and textile vias has been described

196 previously in greater detail [19].
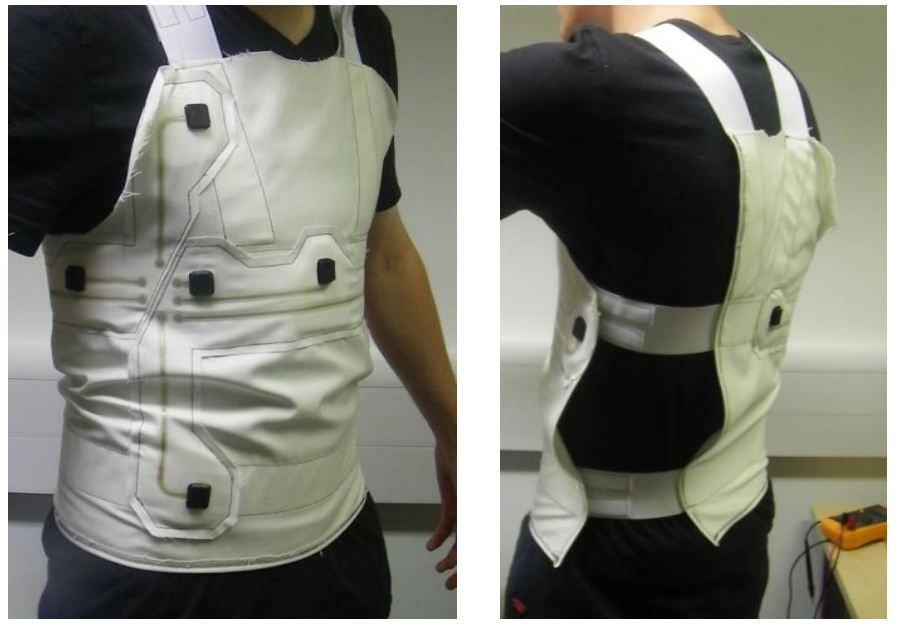

Right - Frank configuration vest worn inside-out viewed from $225^{\circ}$.

178 between the two Lagonda textiles. Elastic strips of width 50

$179 \mathrm{~mm}$ and thickness $1.05 \mathrm{~mm}$ are then attached to improve the

180 contact stability of the electrodes when the vest is worn.

181 Every electrode has an elastic strip over it. These elastic

182 strips have hook and loop textile attached at the ends of the

183 strips so that the vest can be secured around the torso. The

184 complete vest is shown in Fig. 7.

Fig. 8 shows the vest worn inside-out so that the electrode 186 positions can be observed. The final step of the fabrication process involves clamping a steel button, of the type found commonly in garments, through each of the screen printed stencil printing.

Fig. 7. The Frank configuration vest with the screen and stencil printed patches and elastic strips attached. 
The impedance spectrum of the electrodes in this work was

199 examined using a Wayne Kerr 6500B precision impedance

200

201

202

203

204

205

206

207

212 in the $\mathrm{X}, \mathrm{Y}$ and $\mathrm{Z}$ axes as defined in Fig. 1. The resistive

213 network used in this work is unshielded and connects

214 between the vest and the amplifier cables. The value of $\mathrm{R}$, the

215 resistance multiplier in Fig. 10, was chosen as $50 \mathrm{k} \Omega$ in this

216 work to ensure the network resistance is significantly larger

217 than the electrode resistance, as recommended in Frank's

218 original paper [12]. It has been reported that the skin-

219 electrode impedance can exceed even this high value, and

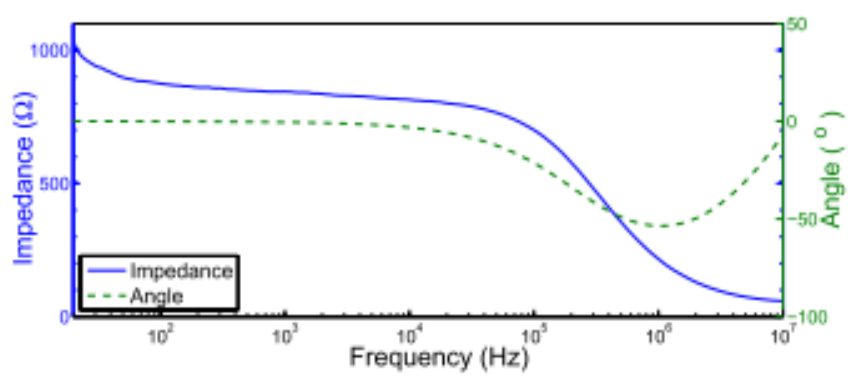

Fig. 9. An impedance/frequency sweep with two textile printed electrodes face to face. No conductive paste is used. Contact pressure is $\approx 25 \mathrm{kPa}$.
220 rubbing or wetting might be required in some circumstances

221 with this prototype.

222 The amplifier circuit used in this work is the

223 instrumentation amplifier circuit described by Spinelli et al

224 which has a gain of 1000 [20]. This amplifier circuit was

225 chosen because it has a high common mode rejection ratio

$226(123 \mathrm{~dB}$ at $50 \mathrm{~Hz})$ and contains an AC-coupling element that

227 rejects DC drift at the electrodes. AC coupling is useful when

228 using dry electrodes that do not have a stable electrochemical

229 potential at the electrode-skin interface, because it rejects

230 some DC drift prior to amplification. A set of these amplifiers

231 in a shielded box were used for this work. All cables other

232 than those printed on to the textile are shielded. The output

233 signals from the amplifiers are digitized using a National

234 Instruments USB-6008 data acquisition device and the

235 signals are recorded and viewed in National Instruments

236 SignalExpress 3.0. The full experimental setup is shown in

237 Fig. 10.

238 The presence of sweat during the recordings improved the

239 performance of the Frank configuration vest due to reduced

240 skin-electrode impedance, while the conventional gel

241 electrodes were more prone to slipping off in sweaty

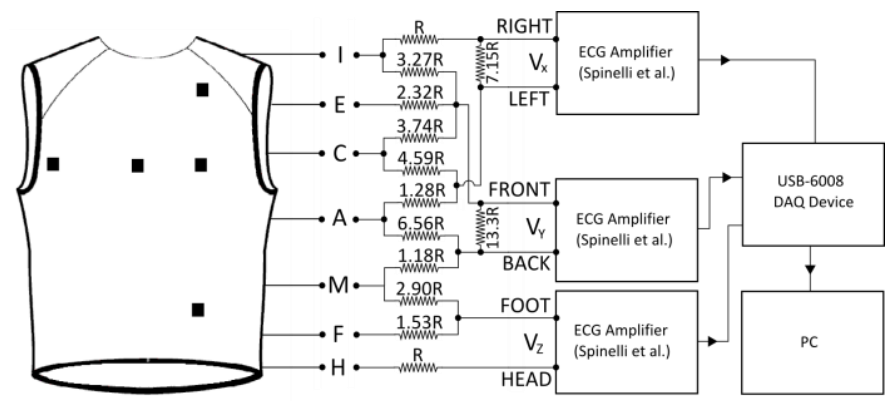

Fig. 10. Full experimental setup for recording signals from the Frank configuration vest. 

wiped dry before each recording. respectively. conditions. To prevent this affecting the results, the skin was

\section{Results}

First, measurements are taken using standard $\mathrm{Ag} / \mathrm{AgCl}$ self-adhesive electrodes. To examine the effect of changing the position of the head $(\mathrm{H})$ and foot $(\mathrm{F})$ electrodes, one measurement is taken with the electrode positions originally proposed by Frank and another is taken with the electrodes on the torso, with the head and foot electrodes in the modified positions. Recordings are taken using 3M RedDot selfadhesive electrodes [21] which are taped to the body and connected to the amplifier with shielded cables. The amplified signals from recordings with the original and modified Frank positions are shown in Fig. 11 and Fig. 12

The main difference between these recordings is observed on the Y signal. In the recordings with the modified positions the amplitude of the peaks on the $\mathrm{Y}$ signal recordings is significantly larger. This is because the Y signal electrodes ( $\mathrm{H}$ and $\mathrm{F}$ ) have been moved on to the torso.

A recording was then taken with the Frank configuration vest. The setup time is under 10 seconds, significantly faster than individually applying electrodes which can take several minutes. No conductive gel was used. The driven right leg (DRL) electrode was not printed in combination with the other electrodes so that different DRL positions could be examined. It was found that the placement and material of the DRL electrode had minimal effect as long as it had stable skin contact, although signal quality appeared to improve with the DRL on the limbs compared to the torso. The DRL
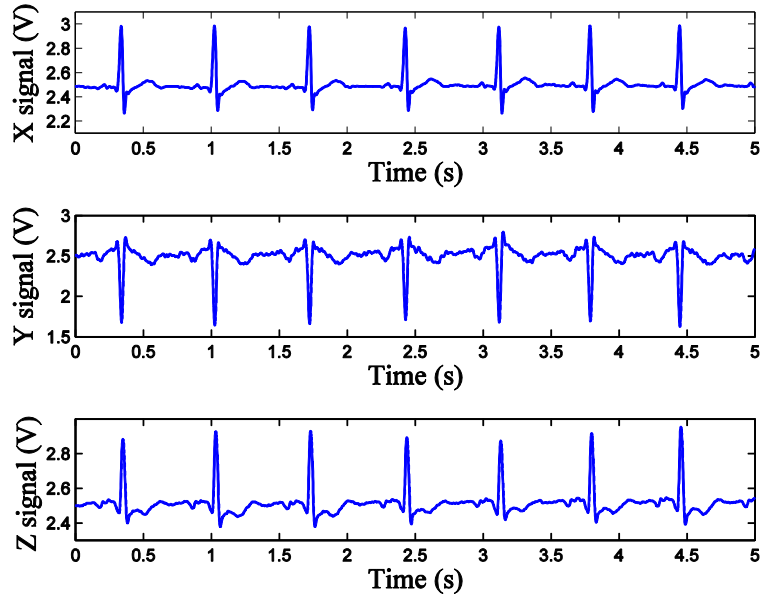

Fig. 11. Amplified signals from Frank configuration recording with the original electrode positions using $3 \mathrm{M}$ RedDot $\mathrm{Ag} / \mathrm{AgCl}$ self-adhesive electrodes.
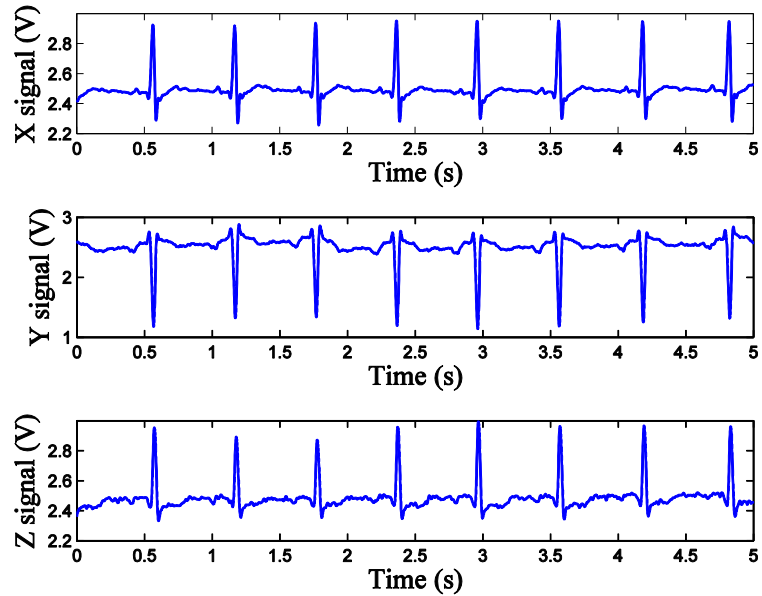

Fig. 12. Amplified signals from Frank configuration recording with the modified electrode positions using $3 \mathrm{M}$ RedDot $\mathrm{Ag} / \mathrm{AgCl}$ self-adhesive electrodes.
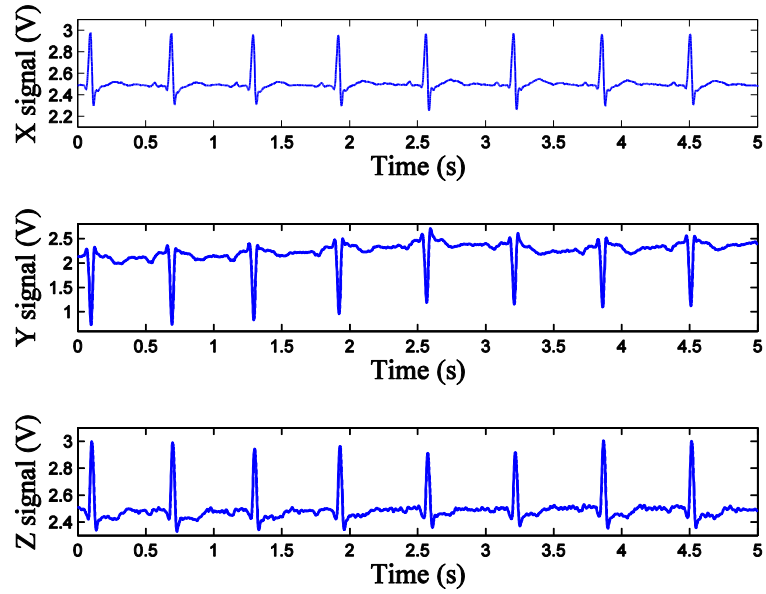

Fig. 13. Amplified signals from Frank configuration recording taken with the screen and stencil printed vest. 
272 electrode used in the recordings shown here was a 3M

273 RedDot solid gel electrode connected to the torso as

274

275 with the vest is shown in Fig. 13.

276 These electrodes match well with the previously recorded

277 modified Frank configuration. There is some $50 \mathrm{~Hz}$ noise on

278 the recordings, notable on the example recording here on the

$279 \mathrm{~V}_{\mathrm{Z}}$ lead. Baseline drift is also more significant, observable

280 here on the $V_{Y}$ lead. There was a settling time of less than

281 five minutes, during which large amounts of baseline drift

282 occurred during motion artefacts. Despite these limitations,

This paper has demonstrated the use of screen and stencil printing to fabricate a prototype Frank configuration monitoring garment. The garment allows the electrical potential from the heart to be monitored in three different axes with a setup time of around 10 seconds and no discomfort.

In a final application several different sizes of the vest would be required to fit various body types because the textile is not stretchable. Increasing the stretchability of the conductive tracks would allow one garment to fit various body sizes and allow for greater skin contact pressure. The fabrication approach outlined here could be used to implement alternative reduced lead configurations, such as
300 the EASI configuration [22], which might be less affected by 301 motion artefacts.

302 The testing method used here is limited, in that there is no 303 numerical analysis of the results or testing on multiple 304 subjects. However, the experiments described here provide a 305 proof-of-concept for this monitoring device and fit within the 306 scope of this paper. With the printed vest there was a settling 307 time of less than 5 minutes due to impedance mismatch while 308 the impedance of the electrodes stabilized. During this 309 settling time motion artefacts and baseline drift were 310 significant and prevented an accurate ECG from being 311 recorded. Even after this settling time some minor motion 312 artefacts were present; although the author could walk 313 without obviously affecting results, the motion artefacts were 314 clear when, for example, an arm was lifted above the head. 315 In practice, this would not prevent diagnostically useful 316 information from being recorded, but would reduce the 317 amount of data available. It would also prevent information 318 from being recorded in any cardiac event that was 319 accompanied by a spasm or a fall. The settling time and the 320 motion artefacts present with passive electrodes can be 321 prevented by using dry active electrodes on woven textile, 322 which have been demonstrated previously by the authors to 323 have no settling time and motion artefact levels as low as 324 clinical Ag/AgCl electrodes [14].

325 The vest reported here can be used to monitor a Frank 326 configuration vectorcardiogram with high quality. After a 327 short settling time the signal noise is low enough that all the 328 ECG deflections, their timing and their relative magnitudes 329 can be observed. The vest is simple to use and has potential 
330 to be used in telemedicine and home health care as it could

331 conceivably be used without specialist training. It also has

332 the potential to save time in hospitals with significantly faster

333 setup procedures.

\section{REFERENCES}

[1] Drake, W.M., Broadhurst P.A., and Dymond, D.S. (1997) Cardiology explained, $1^{\text {st }}$ ed., London: Chapman \& Hall.

[2] Jeffries, P.R., Woolf, S. and Linde, B. (2003) 'Technology-based vs. traditional instruction: A comparison of two methods for teaching the skill of performing a 12-lead ECG.' Nurs Educ Perspect. vol. 24, no. 2, March, pp. 70-74.

[3] Dower, G.E. (1968) 'A lead synthesizer for the Frank system to simulate the standard 12-lead electrocardiogram.' J Electrocardiol. vol. 1, no. 1, pp. 101-116,

[4] Horacek, B.M., Warren, J.W., Stovicek, P. and Feldman, C.L. (2000) 'Diagnostic accuracy of derived versus standard 12-lead electrocardiograms.' J Electrocardiol. vol. 33, no. 1, December, pp. $155-60$.

[5] Nørgaard, B.L., Sørensen, C., Larsen, T., Thygesen, K. and Dellborg, M. (2002) 'Computerized vectorcardiography telemetry: a new device for continuous multilead ST-segment monitoring of ambulatory patients. A preliminary report.' Annals of noninvasive electrocardiology, vol. 7, no. 3, July, pp. 204-210.

[6] Mestrovic, M.A., Helmer, R.J.N., Kyratzis, L., and Kumar, D. (2007) 'Preliminary study of dry knitted fabric electrodes for physiological monitoring.' 3rd IEEE International Conference on Intelligent Sensors, Sensor Networks and Information, Melbourne, pp. 601-606.

[7] Linz, T., Kallmayer, C., Aschenbrenner, R., and Reichl, H. (2006) 'Fully integrated EKG shirt based on embroidered electrical interconnections with conductive yarn and miniaturized flexible electronics.' International Workshop on Wearable and Implantable Body Sensor Networks (BSN'06), pp. 4-7.

[8] Paul, G., Torah, R., Beeby, S., Yang, K., and Tudor, J. (2014) 'A Smart Textile Based Facial EMG and EOG Computer Interface.' IEEE Sensors, vol. 14, no. 2, February, pp. 393-400.

[9] Merritt, C., Carey, R., Nagle, H.T., and Grant, E. (2009) 'Fabric-based active electrode design and fabrication for health monitoring clothing.' IEEE Trans Inf Technol Biomed, vol. 13, no. 2, March, pp. 274-280

[10] Paul, G., Torah, R., Yang, K., Beeby, S., and Tudor, J. (2014) 'An investigation into the durability of screen printed conductive tracks on textiles.' Meas. Sci. Technol., vol. 25, no. 2, January.

[11] Yang, K., Torah, R. Wei, Y., Beeby, S., and Tudor, J. (2013) 'Waterproof and durable screen printed silver conductive tracks on textiles.' Text Res J., vol. 83, no. 19, July, pp. 2023-2031.

[12] Frank, E., (1956) 'An accurate, clinically practical system for spatial vectorcardiography.' Circulation, vol. 13, no. 5, May, pp. 737-749.

[13] Canale, S., Priscoli, F.D., Mignanti, S., Oddi, G., Sassano, A., Macone, D., Piazzo, L., Costa, F., and De Stefanis, P. (2013) 'The Bravehealth Software Architecture for the Monitoring of Patients Affected by CVD', 5th International Conference on eHealth, Telemedicine, and Social Medicine, Nice, pp. 29-34.

[14] Paul, G., Torah, R., Beeby, S., and Tudor, J. 'A Novel Design for Screen and Stencil Printed Active Electrodes on Woven Textiles.' Sensors and Actuators A: Physical, vol. 221, no. 1, January, pp. 60-66.

[15] Kang, T.H., Merritt, C.R., Grant, E., Pourdeyhimi, B., and Nagle, H.T (2008) 'Nonwoven fabric active electrodes for biopotential measurement during normal daily activity.' IEEE Transactions on Biomedical Engineering, vol. 55, no. 1, January, pp. 188-195.

[16] Ottenbacher, J., Romer, S., Kunze, C., Großmann, U., and Stork, W., (2004) 'Integration of a bluetooth based ECG system into clothing.' 8th International Symposium on Wearable Computers, Arlington, pp. 186-187.

[17] Yang, K., Torah, R. Beeby, S., and Tudor, J. (2013) 'Flexible and washable conductive textile achieved by screen printing for smart fabric applications.' Presented at LOPE-C.

[18] Smart Fabric Inks, Fab-Inks, 244 2013, [Online], Available: http://www.fabinks.com/, Accessed [6 6 2015]. 